
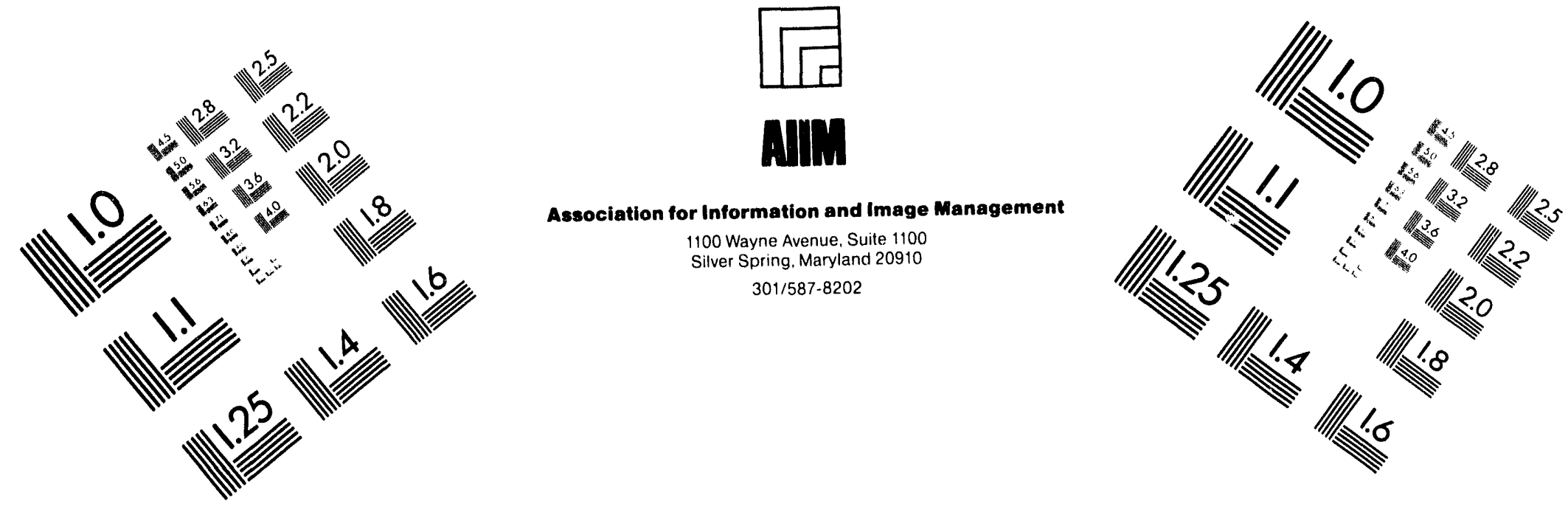

Centimeter

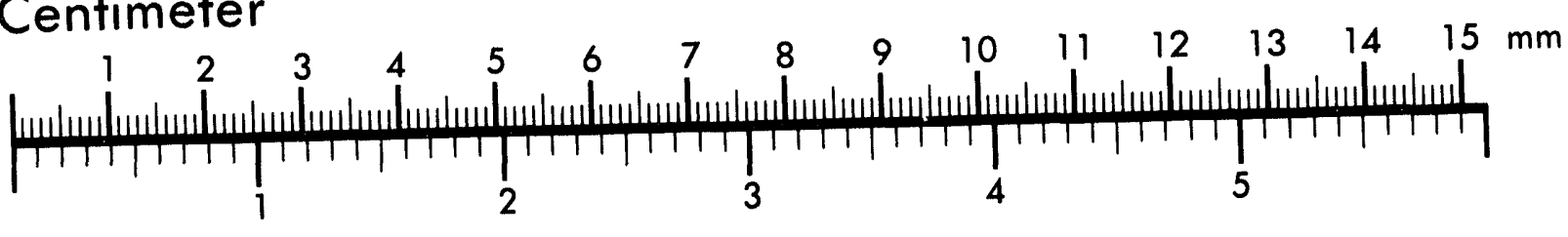

Inches
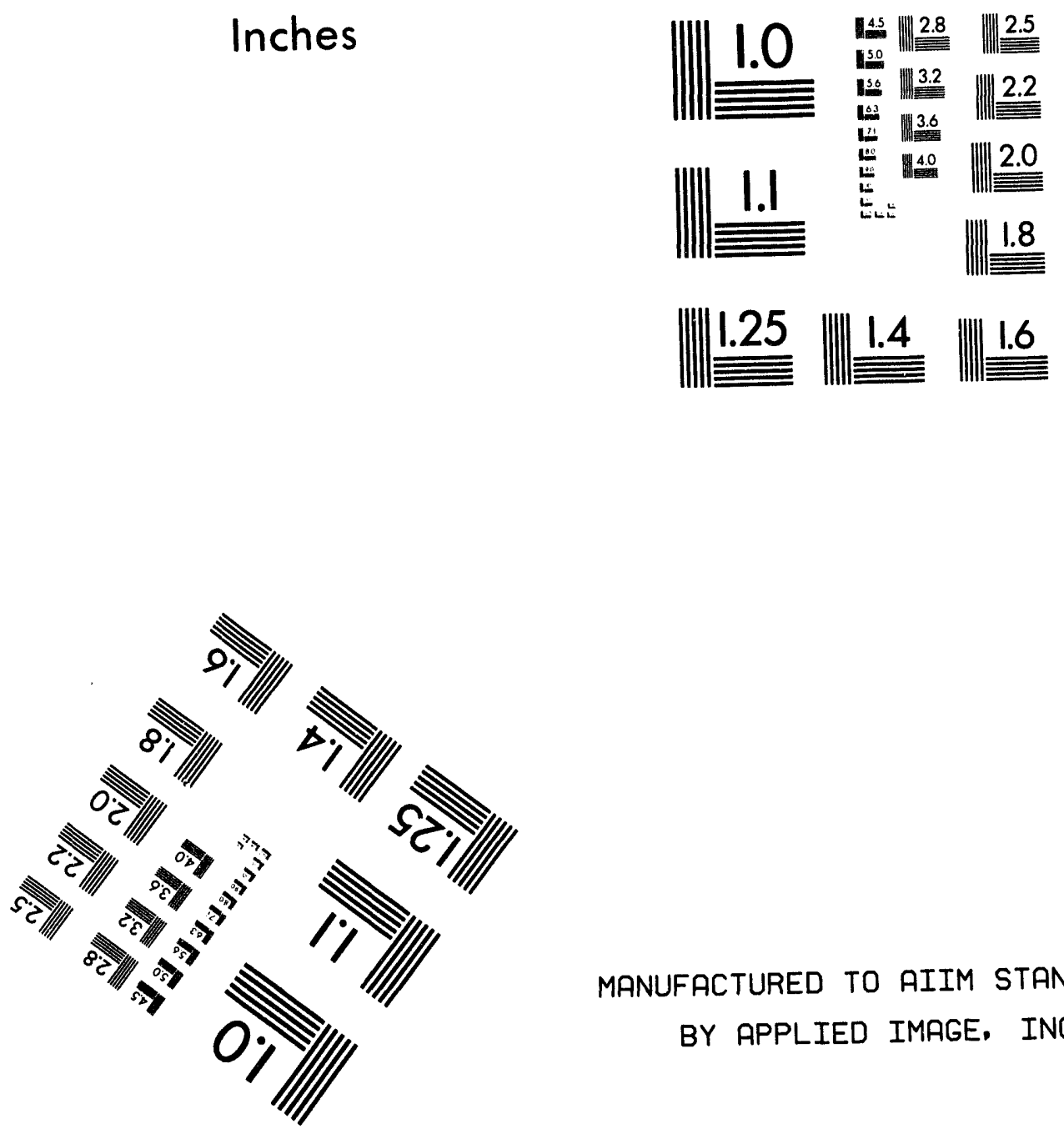

MANUFACTURED TO AIIM STANDARDS

BY APPLIED IMAGE, INC.

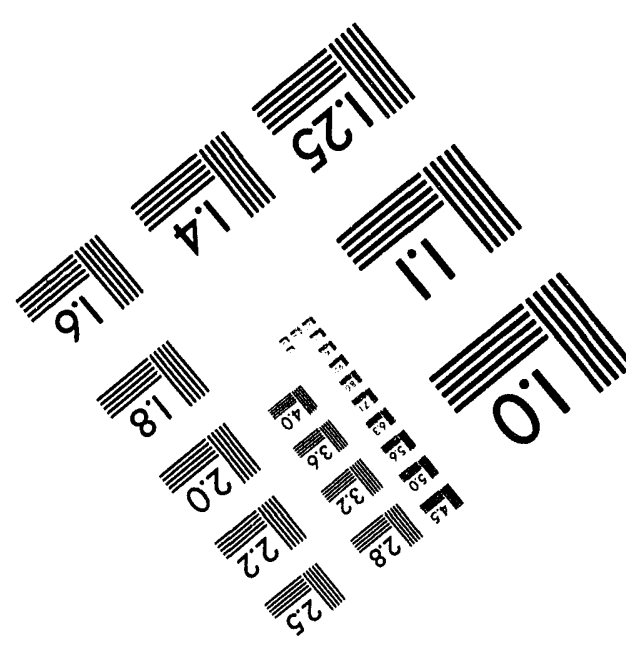



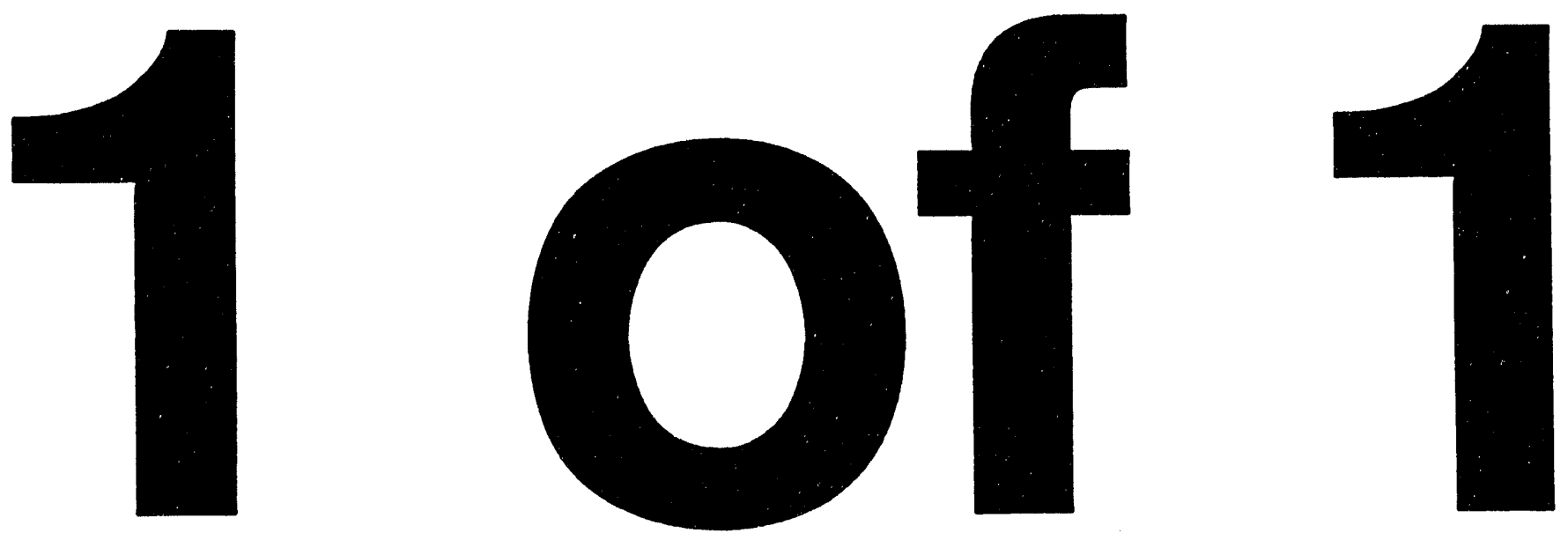
Statistical Process Control Testing Of Electronic Security Equipment

\author{
Dale W. Murray \\ Debra D. Spencer \\ Nuclear Security Center
}

\begin{abstract}
Statistical Process Control testing of manufacturing processes began back in the 1940's with the development of Process Control Charts by Dr. Walter A. Shewart. Sandia National Laboratories has developed an application of the SPC method for performance testing of electronic security equipment. This paper documents the evaluation of this testing methodology applied to electronic security equipment and an associated laptop computer-based system for obtaining and analyzing the test data. Sandia developed this SPC sensor performance testing method primarily for use on portal metal detectors, but, has evaluated it for testing of an exterior intrusion detection sensor and other electronic security devices. This method is an alternative to the traditional binomial (alarm or noalarm) performance testing. The limited amount of information in binomial data drives the number of tests necessary to meet regulatory requirements to unnecessarily high levels. For example, a requirement of a 0.85 probability of detection with a $90 \%$ confidence requires a minimum of 19 alarms out of 19 trials. By extracting and analyzing measurement (variables) data whenever possible instead of the more typical binomial data, the user becomes more informed about equipment health with fewer tests (as low as five per periodic evaluation).
\end{abstract}

\section{TYPES OF DATA}

There are two types of data discussed in this paper, attributes data and variables data. Attributes data are the binomial results of a Bernoulli trial of a go or no-go type of device. Most security equipment fall into this category. Metal detectors and intrusion detectors alarm or do not, and biometric identity verifiers accept or reject the identity of a person requesting admission to a secure area. Most data taken from this equipment in the past have been attributes data.

Variables data are the numeric values that are a measure of some parameter. Magnitude values such as volts, amperes, feet, or other parameters are variables data. For the purpose of our discussion the parameter being measured is indicative of the performance of the device. For instance, a metal detector may have as a performance measurement the voltage of its peak response to a test object. This testing method was first developed at Sandia for use with metal detectors.

\section{Statistical Process Control Theory}

Statistical process control (SPC) is a powerful graphical method of monitoring a process. A close examination of the method and how it relates to performance testing of security equipment will illustrate this method's value in this application.

Using attributes data to evaluate a sensor, that is, to see if its performance meets regulatory requirements, requires much more testing than using a test scheme based upon both attributes and variables data. For instance, using the DOE metal detector testing requirement of a minimum of 0.85 probability of detection at a 0.95 confidence level, a single-phase testing strategy, using attributes data and allowing no missed alarms, requires 19 identically performed tests. A testing strategy allowing one miss (e.g., zero or one miss results in a successful test; two or more missed alarms result in test failure) requires $\mathbf{3 0}$ identically performed tests. Test failure leads to investigation, possible rework, and repeat of detector initiation tests A similar testing strategy allowing up to two misses requires 40 identically performed tests. Clearly, if one is to test several test objects at several locations against the $0.85 / 0.95$ requirements, the quantity of tests quickly becomes too large to be practical. In the case of exterior intrusion detection sensors, the required probability of detection is 0.9 at a 0.95 confidence. Even by restricting the testing strategy to single phase with no misses, the required number of trials is 29 . Facilities may have as many as $\mathbf{4 0}$ sectors so the task of performance testing exterior sensors is very time consuming.

By using variables data in addition to attributes data, we can examine the value of the performance parameter, which is typically the primary alarm variable. From this we can make inferences about probability of detection with significantly fewer tests. This testing establishes a 0.95 confidence interval around the estimate for worstcase passage of the threat object. As long as this confidence interval is above the alarm threshold interval, the detector is working acceptably. This analysis allows assessment of detector variability and investigation of noalarm conditions in a more informed manner.

SPC charts plot periodic averages of peak voltage as well as the range of the values. The process (in our case, the process of metal or intrusion detection over time) is considered to be unchanged (in control) as long as the weekly or biweekly evaluations stay within the $3 \sigma$ control limits plotted on the control charts. The analysis notes other trends, such as extended runs above or below the average, since they could lead to interesting questions about patterns in weekly response.

\section{Control Limits}

The control limits of a SPC chart are calculated and plotted using the following formulas. The averages chart monitors the accuracy of the process (is the average where it should be for proper operation?) For the Averages Chart, 
the upper and lower control limits are given respectively by the formulas

$$
\begin{aligned}
& C L_{U}=\overline{\bar{X}}+0.577 \bar{R} \\
& \text { and } C L_{L}=\overline{\bar{X}}-0.577 \bar{R}
\end{aligned}
$$

where $\overline{\bar{X}}$ is the sum of all sample means divided by the number of samples and $\bar{R}$ is the sum of all sample ranges divided by the number of samples. The Ranges Chart monitors the precision of the process (is the device performing with consistent responses?) The control limits for the Ranges Chart are

$$
\begin{aligned}
& C L_{U}=2.114 \bar{R} \\
& \text { and } C L_{L}=0
\end{aligned}
$$

An estimate of the standard deviation is

$$
\sigma=\bar{R} / 2.3
$$

Good procedure dictates initialization of an SPC chart immediately following an acceptance test (which is not discussed in this paper) where the performance of the detector is deemed acceptable. Periodic testing results plotted on the chart and remaining within the control limits establish considerable confidence that the detector's performance is unchanged since acceptance testing.

\section{Data Collection}

Currently, few types of security equipment are designed with an output port for the explicit purpose of providing variables data. However, several commonly used devices have been tested for compatibly with this method.

\section{Metal Detectors}

Several methods allow variables data acquisition from the various metal detectors. The Infinetics series 500 has, as an option, a liquid crystal display (LCD) voltmeter display. The display device records and displays the detector's peak response for each target passage. Infinetics indicates that it provides a similar interface for the more recent series 700 portal metal detectors.

The EG\&G Astrophysics AT is equipped with a connector located on the back of the electronics unit. This connector provides a parallel output of the analog signal that drives the front panel LED bar meter. The detector outputs this signal on pin 24 of the auxiliary port.

The Outokumpu Metor 120 converts the received signal to a digital format almost as soon as it is received. Because of this, no analog signal is available that is suitable for variables testing. A connector provided for connection of a remote alarm display unit contains an RS-
232 serial output. The serial output contains numeric response data suitable for variables testing.

The Federal Labs Systems Checkgate PC 9000 provides a numerical read-out on a LCD of the detector's response to objects. The numeric response data lends itself well to variables testing.

The Garrett Magnascanner 5000 has an interesting feature that allows variables data acquisition. When in the setup mode a numeric read-out on the LCD is set to the maximum sensitivity setting. The detector then starts resetting this read-out to the level at which it would currently alarm. This resetting process is a one-directional process that seeks the lowest setting that would produce an alarm during the test period. By passing a threat item through the portal in the worst-case location and the worstcase orientation during the test period, the minimum sensitivity setting that would be needed to detect that article is found. This output works well as a variables data source for SPC testing.

\section{Exterior Intrusion Detection Sensors}

To date the only exterior sensor on which Sandia tried using SPC testing is the Racon 14000. Sandia's Exterior Sensor Group selected this sensor as the most likely sensor for successful SPC testing. Test point C (TPC) on the receiver board provides the data chosen for this testing. The analog signal present on this test point outputs a normally high voltage level and responds to stimulus by decreasing in magnitude. Setting the output of TPC at a high level ( 5.5 volts for example) adjusts the sensitivity such that a man-sized stimulus causes this level to drop below 3 volts resulting in an alarm. An automatic gain circuit maintains the level of the output and can cause problems for SPC testing as will be discussed in the section dealing with test results.

\section{Biometric Entry Control Devices}

Althougn this process has not yet been tested on biometric identity devices, efforts to expand this method to these devices are under way. Several performance parameters associated with biometric devices present opportunities for SPC analysis. In many instances, a biometric device produces a repeatable response to standardized test objects. This repeatable response provides data useful for SPC analysis. In this case the SPC analysis monitors the probability of success $\left(P_{s}\right)$ of the biometric identifying a valid request for admission.

The biometric performance characteristics of false reject rate, false accept rate (referred to as error rates), and transaction time provide an even greater opportunity for useful application of SPC analysis. Many biometric devices make available these data for collection during normal operation. Analysis by SPC of these data not only monitors performance but also allows the performance of the biometric to be "fine tuned." Error rate analysis requires considerably more data than other parameters, but this data is obtained inexpensively. Employees must conduct most performance data collection while error rate data is a natural byproduct of normal operation. 


\section{The Repeatable Stimulus}

Mechanical apparatus for carrying a test target through a detector greatly reduces variation in the results (biometrics may have to be handled in a different way). A realistic test object carried by a human subject produces low variability results during testing only if great care is taken to ensure that the object passes through the detector in exactly the same orientation each time. A spherical test object further reduces the variability of the detector response. A geometrically complex object produces a wide variety of response magnitudes when passed through the detector in various orientations; a spherical test object will produce the same response in a sensor regardless of its orientation. Sandia developed a gravity-powered field test track for testing both metal detectors and certain intrusion detectors. The test track operates on the idea that a metallic sphere will roll down an inclined plane with highly repeatable velocity. This track was used while recording data for both the metal detectors and the Racon microwave intrusion detector (see figure 1.)

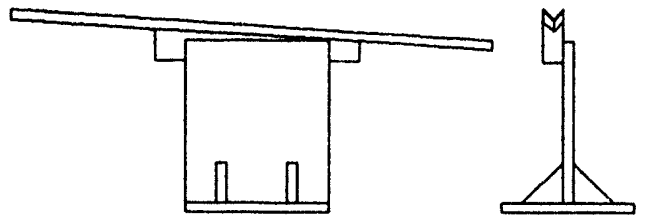

Figure 1. The Sandia Gravity-Powered Test Track

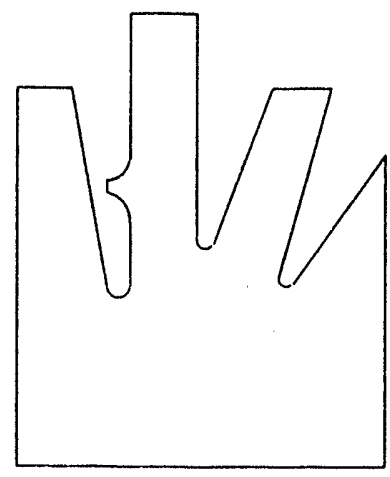

Figure 2. A repeatable stimulus for hand geometry identity verifiers

Sandia is developing a prototype repeatable stimulus test object for a Recognition Systems Handkey (see figure 2.) This hand geometry biometric identity verifier will be among the first biometric identity verifiers to be tested with the SPC method. The biometric process is static (the object being measured does not move) so there is no need for a mechanical means of carrying the test object through the device.

\section{The Statistical. Process Control Computer Program}

The SPC computer program provides the user with a friendly user interface to enter the data into the program and to graphically and numerically analyze the data. The program relieves the user of the task of manually reducing the data and plotting it on the control charts. The program can quickly analyze large blocks of data and produce a high quality graphic output.

The SPC testing sottware is structured into four main functional blocks. The first block is the user interface. The second block is the data entry section. This section must be flexible because there is no standard detector interface or data format. There are both manual, and automated entries with the data being recorded directly by the computer The third functional block of the software is the data reduction and analysis section. This code performs the necessary statistical calculations to perform the statistical analysis. As an example, the control limits are calculated and alarms would be generated here if the detection process is found to be out of control bounds. The final section is the output code where the SPC charts are generated and displayed. Also, data files of the actual variables data can be generated.

\section{The Testing Procedure}

A successful procedure for testing security equipment with SPC will include device characterization testing, initialization or acceptance testing, and periodic reevaluation of the device even though only periodic reevaluation actually uses the SPC program. During characterization testing (which follows initial installation, any movement of detectors, program change, or any rework), the device is tested to determine worst-case combinations of threat object, location, and orientation for detectors and general performance parameters for biometrics. Initialization testing is performed after any characterization testing or when settings are changed. Its primary purpose is to adjust the settings for proper operation. Periodic reevaluation is a more abbreviated testing aimed at periodically reestablishing that operational behavior has not been altered since the last initialization testing.

The use of variables data allows us to learn more about the operation of the equipment while using less data. Howtver, a need for consistency between trials becomes even more important when using variables data, although the same need exists whether using attributes or variables data. During any group of trials that will be considered identical in statistical analysis, variability must be kept to a minimum. Sources of possible variability in detector test data include:

- changes in metal detector location (metal detectors)

- changes in location of large metallic objects nearby (metal detectors and intrusion detectors) 
- test-object path through the detector (metal and intrusion detectors)

- test-object orientation (metal and intrusion detectors)

- changes in test objects (metal and intrusion detectors)

- external electromagnetic interference (metal and intrusion detectors)

\section{Characterization Testing}

Characterization testing determines worst-case combinations of threat object, location, and orientation for a particular detector. In the case of biometrics, it determines likely false accept rates, false reject rates, and transaction times. In addition, studies of response-to-testobject characteristics help approximate operational threshold or sensitivity settings. At the time of characterization testing, the equipment has already been installed in its final operational location. The results of this testing also provides information on how to perform tests to obtain variables data suitable for SPC analysis. The results of the characterization testing is used in initialization testing, which is the next phase of the procedure.

\section{Initialization or Acceptance Testing}

The purpose of initialization testing is 1) to determine the appropriate sensitivity or threshold setting for the installed device to meet regulatory requirements, 2) to establish the detector alarm threshold interval, and 3) to prepare for future periodic testing. Initialization testing is performed after any characterization testing and any time the operational settings are changed.

Initialization testing starts with realistic testing using human test subjects and concludes with mechanical testing aimed at establishing a baseline for comparison during periodic reevaluation. All mechanical test results will be collected, summarized, and displayed by the laptop computer.

The use of SPC charts during initialization has two purposes: to determine process capability; and to initialize the control charts and determine the values to be used as $3 \sigma$ control limits. Experts generally agree that testing subgroups of 5 trials is acceptable. In most cases a minimum of 50 trials is recommended to determine process capability, which includes 1) calculating control limits, and 2) determining if any initial data indicate a process that is stable, in control, and capable of being tracked with SPC. Whether or not the process values lie within product specifications or, in this case, meet regulatory requirements has already been established through realistic testing.

To fully initialize the control charts, you will need approximately 25 subgroups of five tests each to minimize the impact of any extreme values. Conducting 125 tests is not usually convenient in one session, and the process itself may have some natural time variation. Because of this the initialization can take place over the first few weeks. Fifty trials can be done initially, followed by five weeks of 15 trials each. At the end of this time, the process capability study is updated, and initialization is considered complete if the results are satisfactory. During the five-week study period, any values outside the trial control limits should lead to a repetition of the realistic two-step acceptance test described above. Afterward, periodic reevaluation (see next section) will track metal detector operation.

Biometrics testing for error rates will require considerably more trials because the expected rates may be as low as $1 \%$ to $2 \%$. Again, this data is easily and quickly obtained as a natural byproduct of normal operation. Biometric error rates can be plotted using this program with some prereduction of the data. The data must be divided evenly into sufficiently large groups to include some false accepts or false rejects. The size of these groups is determined by the error rates of the specific biometric. The data is reduced by dividing the number of errors by the number of trials in the group. This reduced data point will then be treated by the program as a single trial.

If the process does not seem capable of being in control, resort to realistic testing but continue to collect variables data in addition to the attributes data and use the laptop to generate charts. Experts may be able to help you analyze your data and improve your process so that it can be controlled through SPC charts.

\section{Fariodic Reevaluation}

After initialization testing is completed, weekly periodic reevaluation will test samples of 5 trials using the same mechanical testing procedure used at the end of detector initialization. For biometrics, response to a standardized test object as well as reduced error rate data may be used. The laptop computer will continue to be used to record results, and to plot a new SPC chart including the latest data. The only outputs from routine periodic reevaluation are new SPC charts.

By observing values on the control charts, one has an indication that the process of detector operation has changed. Test group averages that fall below the lower $3 \sigma$ control limit or ranges above the upper $3 \sigma$ are indications that one should determine if there is a problem that requires attention. SPC charts are traditionally doublesided with upper as well as lower control limits. This is important in manufacturing processes and, in fact, is also important in performance testing. With the detection process, exceeding the upper control limit is an indication of a sensitivity that is unnecessarily high which could result in excessive false or nuisance alarms. This does not indicate, however, that the detector will not perform its intended function. For these reasons, exceeding the upper limit may not be considered as serious as exceeding the lower limit. Observations of any changes in environment or procedure and a repeat of the realistic acceptance test are sufficient reaction. If the detector fails the realistic acceptance test, diagnostic-analysis and corrective-action are required.

In addition to values falling outside the control limits on the averages or ranges charts, one should observe and react to nonrandom events such as runs, where several 
successive values are on the same side of the mean, and trends. These events are automatically detected and flagged by the SPC computer program. The progran monitors for occasions when:

- two of three points fall on the same side of the mean and more than $2 \sigma$ away from the mean,

- four of five points fall on the same side and more than $1 \sigma$ away from the mean,

- and eight successive points fall on the same side of the mean.

For normally distributed data, an interval of $\pm 3 \sigma$ about the mean contains $99.7 \%$ of all the values. Thus one could expect that approximately 3 out of 1000 tests would result in values falling outside the control limits, even if no change has occurred. As a result, SPC charts will occasionally indicate a problem when none exists.

When the process really has changed, the SPC charts will need to be reinitialized. This does not always mean that the sensitivity settings of the detector will need to be changed. For instance, the testers may have become more consistent with their test methods. Reinitialize the SPC charts when:

- the process changes (new subgroups do not fit in with old ones),

- initial limits were trial ones, or

- there is some indication of inflated limits such as 1) the presence of range values beyond the upper control limit or 2 ) the average range exceeds $2 / 3$ of range values. In this case median ranges could be used instead of average ranges (the SPC program does not currently support the use of median ranges).

\section{TEST RESULTS}

This section contains the results of tests performed on four portal metal detectors and a Racon 14000 bistatic microwave detector. The figures presented in this section are the actual output of the SPC computer program. Not all of the data taken is presented; the figures are selected as examples of the various testes that were performed.

\section{Metal Detector Basic Control}

The first tests performed were to determine if four metal detectors could remain in control (stay within the control limits) under normal operating conditions. In all cases the detectors performance stayed within control limits throughout the testing.

\section{Static Metal Interference}

The detectors were tested for SPC chart sensitivity to static metal interference. Although static metal in the vicinity of the metal detector does not cause false alarms, it does cause regions of lower sensitivity in the detector.

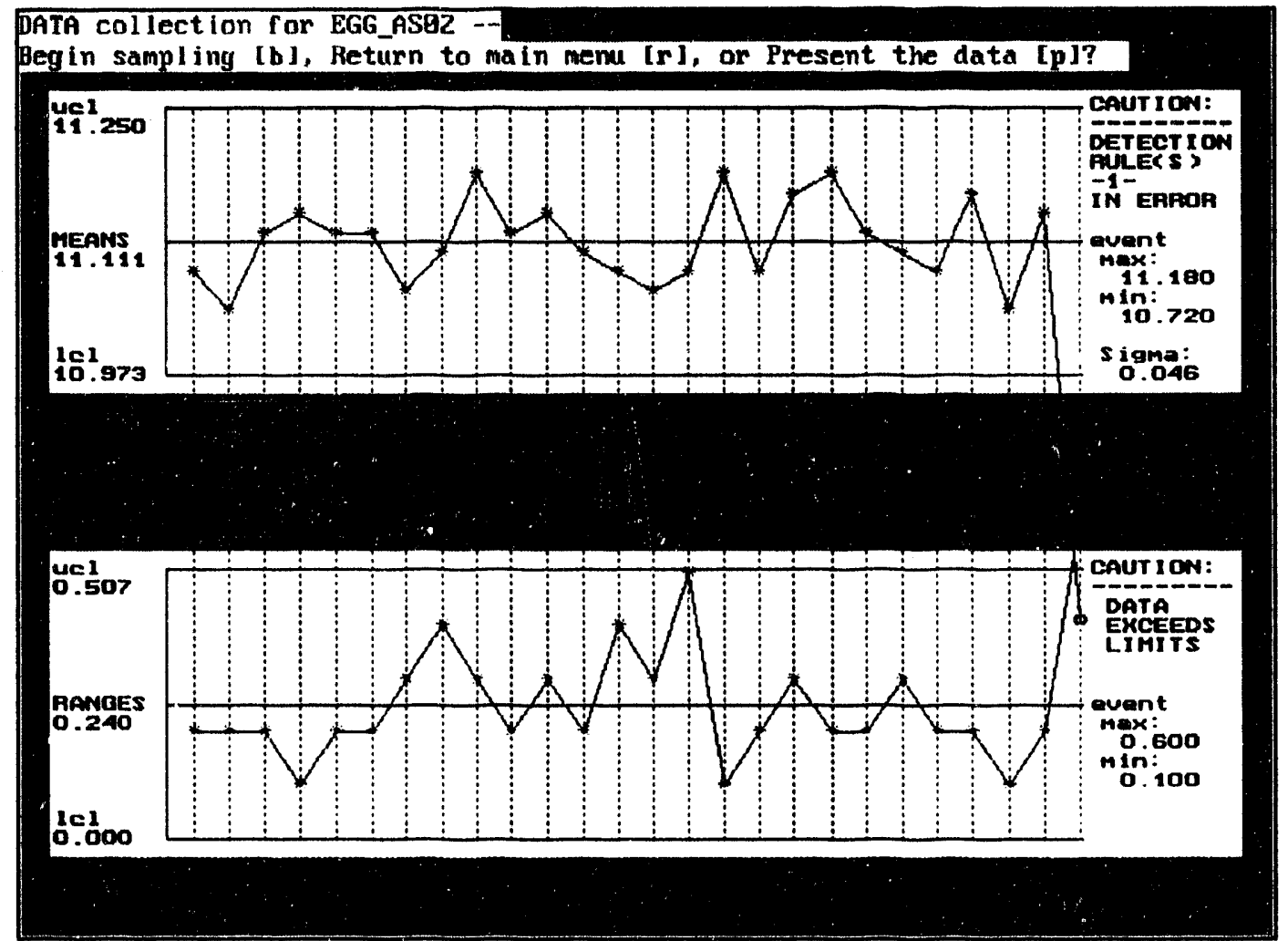

Figure 3. SPC data from an EG\&G/Astrophysics AT with "in-control" initialization followed by intentional static metal interference 
Placing an aluminum plate near the side of the detector simulates the inadvertent placement of other equipment or items such as filing cabinets or metal trash cans too near the detector. This change in the detector's environment would cause the detector to fail the traditional weapon tests. If the SPC testing were to be as effective as the traditional test, it should register a clear indication that something has changed in the detector's performance with a single test. Figure 3 shows that the SPC testing is sufficiently sensitive to the change to detect the change on the first test. Although only the results for the EG\&G/Astrophysics AT are shown, the test was positive for each detector on the first trial.

\section{Tamper}

Making small adjustments to the detector sensitivity and using the SPC program to try to detect the change determined the SPC method's sensitivity to device tampering. Any changes in sensitivity that were large enough to cause a detector to fail the traditional weapons testing procedure should result in an out-of-limits alarm on the first trial using SPC testing. Again the results showed that for all the detectors, the SPC testing is sensitive enough to detect tamper on the first trial.

\section{Racon}

The testing that was performed on the Racon intrusion detector was to determine if the SPC testing method could be used for testing this type of sensor. In fact the results are somewhat inconclusive. Although variables data could be taken from the sensor and entered into the SPC program, all attempts to initialize the control chart with the sensor "in control" failed. There are several reasons for this failure. Because this sensor is located outside, the sensor is subject to many more variations in its environment. These variations in environment influence the detector's response to the point that SPC testing with the current program is very difficult at best. Another compounding factor in the testing process is that the detector responds to the person who is performing the test. Great care must be exercised when testing this detector to not allow the detector to "see" the person performing the test. And finally, the receiver is equipped with an automatic gain control (AGC) circuit that adjusts to try to maintain its adjusted signal level. The very act of sending the test target through the sensor disturbs the AGC, and the signal level does not return to normal for nearly a minute.

The first attempt for initialization is shown in figure 4. Actually this data stayed "in control" throughout most of the initialization period. In the first and middle sections of the data there are obvious downward trends in the values of the subgroup means (note: the Racon has an inverted scale- smaller numbers indicate higher sensitivity). The reason for this trend is that the data was taken at too great a rate. The test sphere was sent down the test track before the AGC had time to recover. The region of higher sensitivity in the latter portions (where the control limit is exceeded) occurred in a period when data was recorded at a much slower rate.

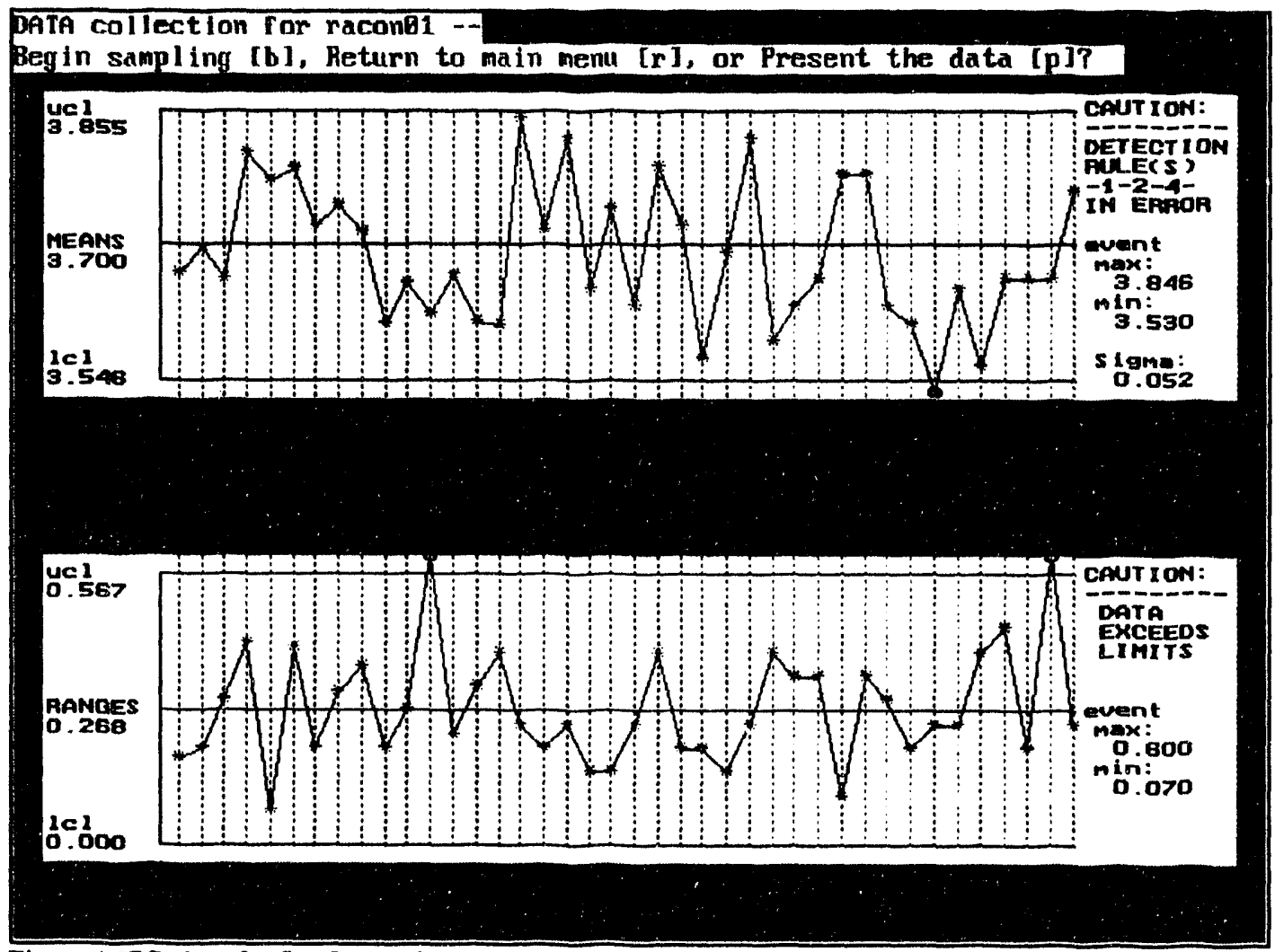

Figure 4 SPC chart for first Racon data 
Thus, when recording data from the Racon, the data acquisition rate can only be about one sample per minute. A second attempt to initialize the Racon ended with similar results. Having learned that the data must not be taken at such a fast rate, the second set of data was taken at the one sample per minute rate. Despite the slower rate, the data did not stay in control. The second attempt revealed that the Racon is sensitive to weather conditions. This data was taken over a period of several days when the wind was gusting. It appears that the Racon responds to the wind (probably because the wind was moving a nearby chainlink fence) with reduced sensitivity. The sensor had been tested thoroughly to ensure its probability of detection. The problem, therefore, was not that the response was inadequate but rather the SPC testing failed to establish realistic $3 \sigma$ limits. The reason for this is that large groups of data were taken in relatively short periods of time. The typical method of initializing the SPC chart by performing 50 immediate samples may not work well with this sensor or perhaps any exterior sensor. If the initialization data is taken in smaller groups over time, the data may include the response over a wider range of environmental conditions. Wider responses during the early phases would result in more realistic $3 \sigma$ limits.

\section{CONCLUSIONS}

All the test results indicate that SPC testing can be successfully used for field performance testing of portal metal detectors. The testing also indicates that this testing methodology is very sensitive to changes in the performance of metal detectors caused by tampering with the sensitivity and changes in the detector's environment.

Although all tests performed on the Racon failed to stay in control with the existing program, there is still great promise for testing exterior intrusion detectors using Statistical Process Control testing methods.

\section{TECHNICAL REFERENCES}

Donald J. Wheeler and David S. Chambers. Understanding SPC, 2nd ed. SPC Press, Inc., 1992

Roger W. Berger and Thomas H. Hart. Statistical Process Control, A Guide for Implementation. Marcel Dekker, Inc., American Society for Quality Control, 1986

A. V. Feigenbaum. Total Quality Control, 3rd ed. New York: McGraw-Hill, 1983

Eugene L. Grant and Richard S. Leavenworth. Statistical Quality Control. McGraw-Hill Co., 1972

Thomas Pyzdek. Pyzdek's Guide to SPC. Volume Two, Applications and Special Topics. ASQC-Quality Press, 1992

Gerald Smith. Statistical Process Control and Quality Improvement. Macmillan Publishing Company, 1991
This work was performed by Sandia National Laboratories under Department of Energy contracts DEAC0476DP00789 and DE-ACO494AL85000.

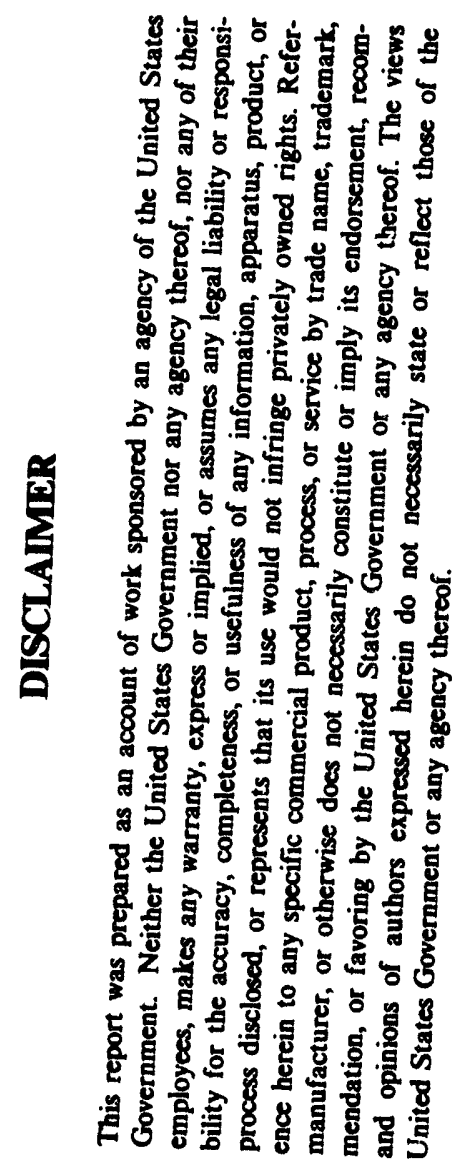



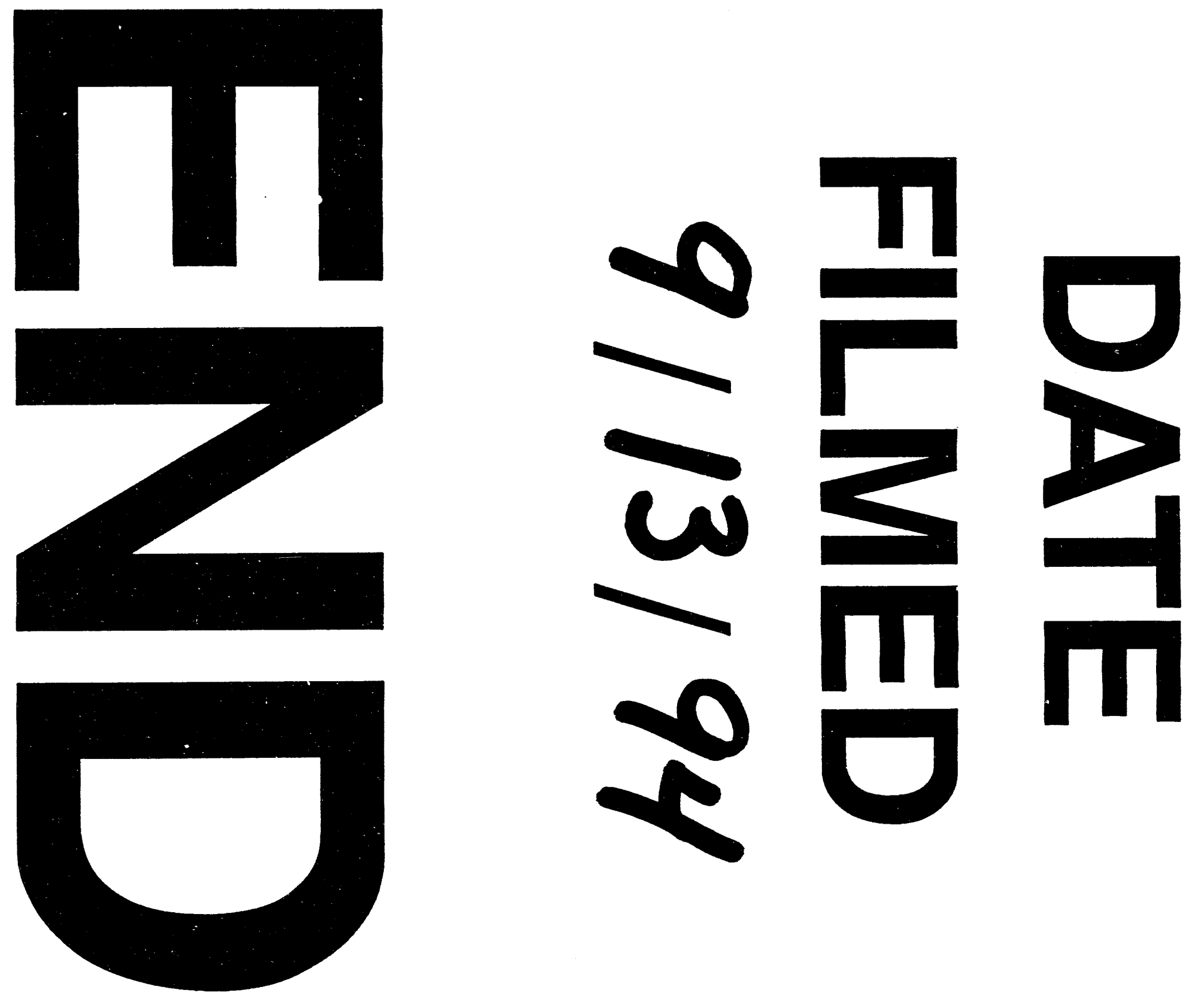


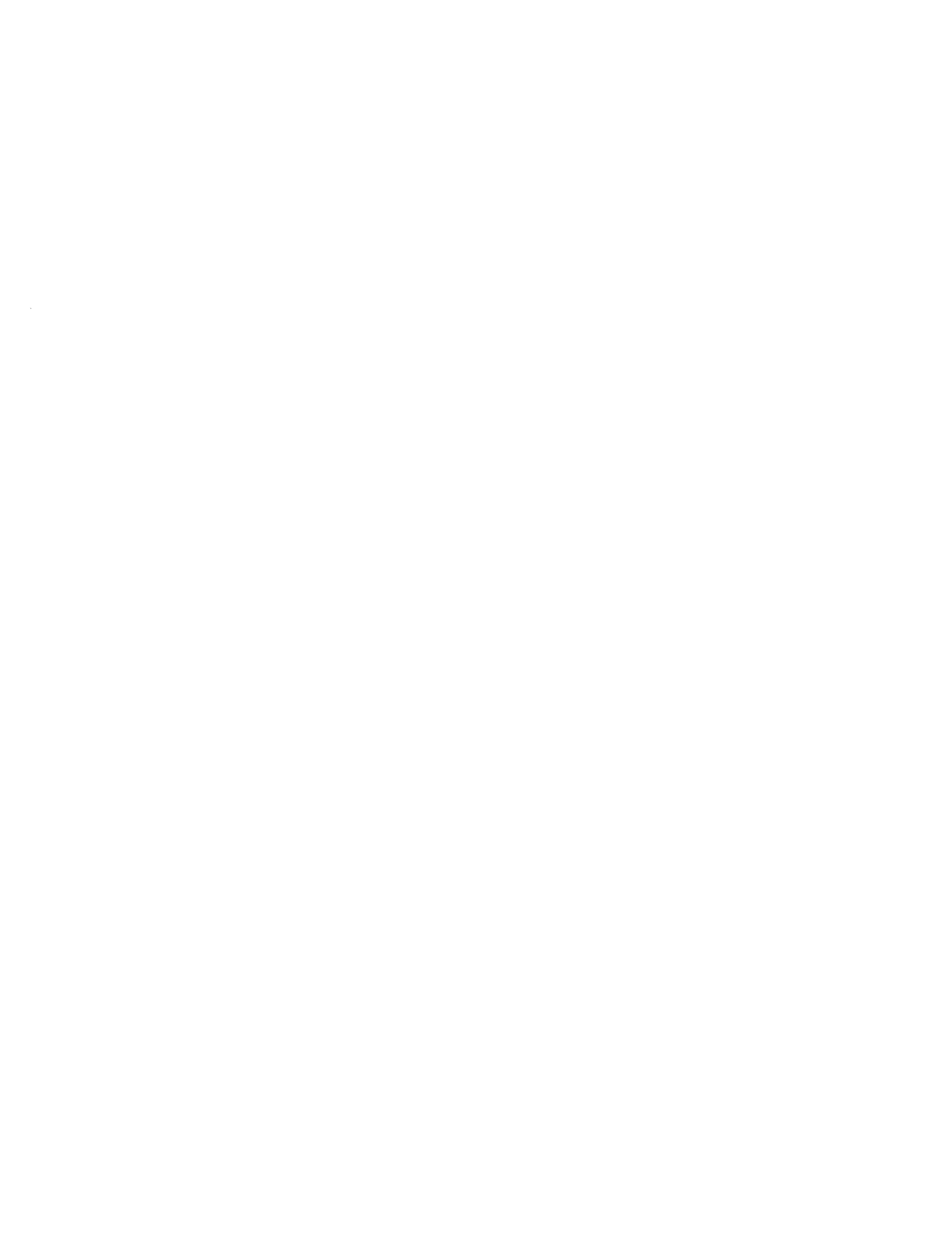

